\title{
Die Behandlung der lebensbedrohlichen Beckenverletzung*
}

\author{
T. Pohlemann, A. Gänsslen
}

\section{Zusammenfassung}

Beckenverletzungen sind seltene Verletzungen, die nur etwa 3\% aller Frakturen umfassen, aber durch ihre noch hohe Letalität ( $8 \%-20 \%)$ eine besondere Rolle als „Problemverletzung“ einnehmen. Die Prognose der Beckenverletzung hängt im wesentlichen vom begleitenden pelvinen Weichteilschaden und dem Grad der Beckenringinstabilität ab. Das „komplexe Beckentrauma“ definiert als Beckenfraktur mit zusätzlichen pelvinem Weichteilschaden definiert (Haut, Weichteile, Darm, Urogenitaltrakt) umfaßt zwar nur etwa 10\% aller Bekkenverletzungen, ist aber durch eine signifikant erhöte Letalität von den
Frakturen ohne signifikanten Weichteilschaden abgesetzt. Aufgrund des hohen Blutverlustes kommt der Akutbehandlung der mechanisch instabilen Beckenverletzung mit begleitender Kreislaufinstabilität eine besondere Bedeutung zu. Die Haupttodesursache derartiger Verletzungen ist in der Frühphase der Verblutungstod, in der Spätphase Schockfolgeerkrankungen wie ARDS und Multiorganversagen. Nur eine sofortige Blutstillung und aggressive Schockbehandlung kann die Prognose dieser Verletzungen verbessern. Anhand eines Notfallalgorithmus wird das Management dieser seltenen Verletzungskombination (maximal $1-3 \%$ aller Beckenfrakturen) dargestellt.

\section{Einleitung}

Die Schwere einer Beckenverletzung wird während der Notfallversorgung häufig erst verzögert erkannt. In den meisten Fällen ist der Weichteilmantel geschlossen, so dass das äußere Erscheinungsbild der hochgradig instabilen, lebensbedrohlichen Beckenverletzungen eher unauffällig erscheint. Ausgedehnte Einblutungen in die Weichteile mit Veränderungen der äußeren Kontur können hier erste Hinweise auf pelvine Blutungen geben. Lediglich bei den seltenen, offenen Beckenzerreißungen finden sich „spektakuläre“ klinische Bilder mit externer Massenblutung und grotesken Fehlstellungen. Da instabile Beckenringverletzungen in über $80 \%$ der Fälle mit weiteren schweren Begleitverletzungen kombiniert sind, wird das kom-

\footnotetext{
* Die Abbildungen 1, 4, 5, 7, 8a und b wurden entnommen aus Tscherne: Unfallchirurgie; Tscherne, Pohlemann: Becken und Acetabulum. Springer Heidelberg 1998.
}

OP-JOURNAL 2000; 16: 268-274

(C) Georg Thieme Verlag Stuttgart · New York plette Ausmaß der Beckenverletzung oft erst dann erkannt, wenn der Blutverlust Ausmaße erreicht hat, die zum unmittelbaren Eingreifen zwingen.

Um diese Patientengruppe zu identifizieren, haben sich in den letzten Jahren die folgenden Definitionen als sinnvoll und brauchbar erwiesen, um Letalität und Gesamtprognose verschiedener Verletzungsarten unabhängig von der Klassifikation der osteoligamentären Verletzung, abschätzen zu können:

\section{Definitionen}

1. „Unkomplizierte“ oder „einfache“ Beckenfraktur: Beckenrand- und Ringbrüche ohne wesentlichen begleitenden peripelvinen Weichteilschaden (Wiederherstellung der osteoligamentären Integrität steht im Vordergrund)

2. Komplexe Beckenfrakturen [1]: Beckenrand- oder Beckenringfrakturen mit begleitendem peripelvinen Weichteilschaden (Gefäße, Nerven Urogenitalverletzungen, Darmverletzungen, Haut- und Weichteilverletzungen).
3. Offene Beckenfraktur: Komplextrauma mit Eröffnung der Haut oder von Hohlorganen (Rektum, Vagina)

4. Traumatische Hemipelvektomie: Abrissverletzung einer Beckenhälfte mit Durchtrennung der großen Gefäßund insbesondere der Nervenbahnen (selbst bei Extremitätenerhalt ist eine Funktionserholung nicht zu erwarten).

Im Folgenden soll das Management für Patienten mit instabilen Beckenringfrakturen und begleitender, beckenbedingter Kreislaufinstabilität dargestellt werden. Diese Patienten sind der Untergruppe „Komplextrauma“ des Beckens zuzuordnen. Die komplexe Beckenverletzung ist dabei mit einer Häufigkeit von ca. 10\% eine eher seltene Verletzung [6]. Nur etwa $1-2 \%$ aller Beckenfrakturen gehen mit akut lebensbedrohlichen pelvinen Blutungen einher. In diesen Fällen kann nur eine sofortige Intervention unter Anwendung eines prioritätenorientierten Diagnostik- und Therapiekonzeptes das Leben des schwerstverletzten Patienten retten. Selbst bei einer ausgezeichnet funktionierenden Rettungskette mit optimaler Therapie am Unfallort, auf dem Transport und in Schwerpunktzentren liegt die Letalität nach komplexen Beckenverletzungen noch bei $20 \%$ bei Transfusionspflichtigkeit sogar bei 33\% [7].

\section{Praeklinisches Management}

Bereits präklinisch ergeben sich erste Hinweise auf das Vorhandensein und die Schwere von Beckenverletzungen. Da offensichtliche Verletzungszeichen häufig fehlen, stützt sich die Diagnose im Wesentlichen auf die Kenntnis des Unfallmechanismus.

Der Unfallmechanismus ist präklinisch häufig der einzige Hinweis auf eine schwere Beckenverletzung.

Nach Hochrasanztraumen und bei Mehrfachverletzten muss immer mit Beckenfrakturen gerechnet werden. Bei poly- 
traumatisierten Patienten bestehen in etwa bei $1 / 4$ der Patienten zusätzliche Beckenverletzungen. Klinisch kann v.a. die Beckenstabilität beurteilt werden. Dabei werden vier klinische Grade der Beckeninstabilität unterschieden [8]:

- Stabiler Beckenring

Beckenringinstibilitäten lassen sich klinisch in vier Schweregrade unterteilen.

Sowohl bei der anterior posterioren Kompression auf den Beckenkamm als auch bei der seitlichen Kompression der Beckenkämme lässt sich keinerlei Bewegung auslösen („der Beckenring ist knöchern fest).

\section{Federnd instabil}

Bei Instabilitäten des Typ B, oft bei Innenrotationsverletzungen, lässt sich bei anterior posteriorer und seitlicher Kompression eine federnde Bewegung innerhalb des Beckenringes feststellen. Dieser Befund ist besonders bei adipösen Patienten leicht zu übersehen und benötigt Erfahrung in der Interpretation. Die Stabilität des Beckenringes ist nicht wesentlich beeinträchtigt.

\section{Ausgeprägte Rotationsinstabilität}

Besonders bei anterior posteriorer Kompression „klappen“ die Beckenhälften weit auseinander und lassen sich bei seitlicher Kompression wieder schließen bzw. sogar überkomprimieren. Eine Translation der Beckenhälfte im Sinne einer Verschiebung nach posterior oder kranial lässt sich aber nicht auslösen.

\section{- Komplette Instabilität (Translations- instabilität)}

Hier lässt sich bei anterior posteriorer Verschiebung, bei seitlicher Kompression und bei Zug oder Stauchungsbewegungen am Bein eine zum Stammskelett unabhängige Bewegung der Beckenhälfte provozieren. Dieser Tastbefund entspricht einer Verletzung des Typs C mit translatorischer Instabilität des hinteren Beckenringes (Blutungsrisiko!).

Klinische Zeichen der Beckeninstabilität und des Vorliegens eines Komplextraumas sind äußerlich sichbare Blutungen, Fehlstellungen und offene Frakturen.

Besteht eine externe Massenblutung, wird durch direkte manuelle Kompression auf die Wunde eine Reduktion der

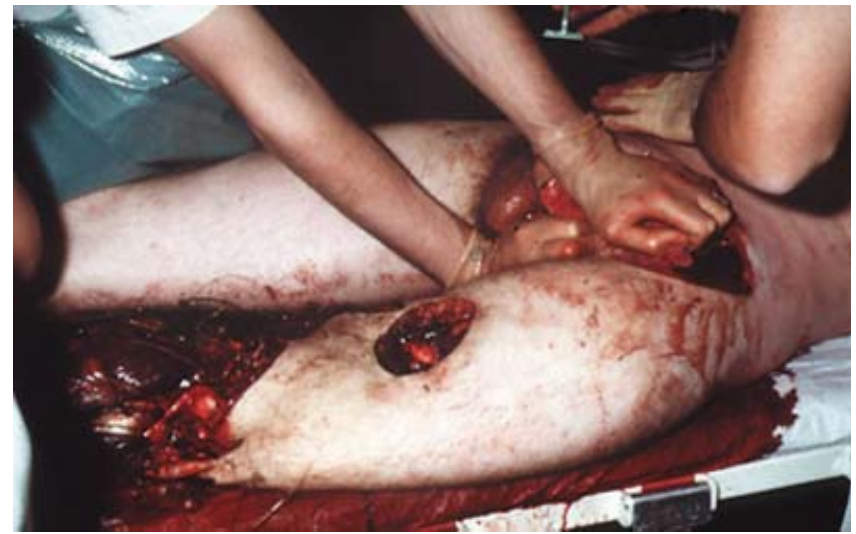

Abb.1 Bei externer Massenblutung kann durch direkte manuelle Kompression auf die Wunde eine Reduktion der Blutungsstärke bzw. eine Blutstillung erreicht werden.

Blutungsstärke bzw. eine Blutstillung erreicht (Abb.1). Diese Patienten müssen auf schnellstem Wege unter forcierter Schocktherapie in das nächstgelegene operationsbereite traumatologische Schwerpunktzentrum gebracht werden („Load and go“).

Kreislaufinstabile Patienten müssen unter forcierter Schocktherapie umgehend in die Klinik gebracht werden.

Für den Transport wird der Beckenverletzte auf einer Vakuummatratze gelagert. Besteht eine hochgradig instabile Außenrotationsverletzung mit sichtbarer Fehlstellung und in der manuellen Prüfung feststellbarer Instabilität, wird schon primär eine Reposition unter Längszug des betroffenen Beines mit Innenrotation und seitlicher manueller Kompression durchgeführt. Dadurch wird das Volumen im kleinen Becken verringert und so eine gewisse Blutstillung erreicht.

Eine Notfallreposition kann durch Rotation und Längszug an der betroffenen Extremität erreicht werden.

Diese Stellung wird durch Anformen der Vakuummatratze gesichert. Aushilfsweise kann die Lagerung durch Umschlingung eines Tuches um Becken und Beine gesichert werden. Schon beim klinischen Verdacht einer Beckenringinstabilität sollte eine erweiterte Schocktherapie begonnen werden.

Der Transport des beckenverletzten Patienten erfolgt möglichst schonend, bevorzugt im Rettungshubschrauber und immer mit Arztbegleitung.

\section{Klinisches Management}

Das klinische Management orientiert sich an einem Notfallalgorithmus. Ein Notfall- algorithmus sollte verschiedene Anforderungskriterien umfassen. Die Eingangskriterien sollten eindeutig und klar sein, er sollte möglichst wenige und klare Entscheidungsmöglichkeiten umfassen und die Begleitverletzungen sollten durch Integration in den allgemeinen Polytraumaalgorithmus berücksichtigt werden.

Basierend auf diesen Kriterien wurde die Entscheidungsfindung bei instabilen Beckenverletzungen mit instabilen Kreislaufverhältnissen auf drei Entscheidungen innerhalb der ersten 30 Minuten nach der Einlieferung reduziert. Die wichtigsten Ziele sind die Lokalisation der Blutungsquelle, die Bestätigung der Beckenringinstabilität und die Beurteilung der Reaktion des Kreislaufs auf Volumensubstitution und Beckenstabilisierung. Eine Embolisationsbehandlung ist dabei nicht primär in den Entscheidungsablauf integriert, da nur arterielle Blutungen embolisierbar sind und deren Anteil als Blutungsursache bei schweren Beckenverletzungen auf lediglich 10-20\% der Fälle geschätzt wird [4]. Die wesentlich häufiger zu beobachtenden venösen Blutungen werden somit nicht gezielt therapiert.

Pelvine Blutungsursachen sind in $80-$ $90 \%$ venöse Blutungen. Nur in $10-20 \%$ liegen Verletzungen großer arterieller Gefäße vor.

\section{Notfallalgorithmus (Abb.2)}

Die erste Entscheidung fällt direkt nach Einlieferung. Liegt eine externe pelvine Massenblutung vor, wie z.B. bei traumatischer Hemipelvektomie, oder nach schwerem Überrolltrauma im Sinne einer „Crushverletzung“, wird der Patient unverzüglich unter forcierter Blutsubstitution zur operativen Blutstillung in den Operationssaal gebracht. 




Abb. 2 Notfallalgorithmus Becken.

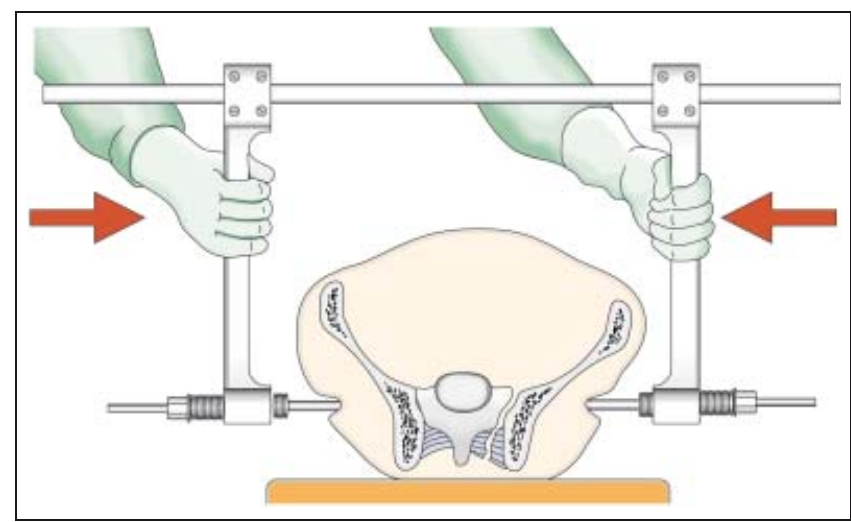

Abb.5 Die Beckenzwinge wird durch manuelle Kompression an den Spannarmen positioniert.

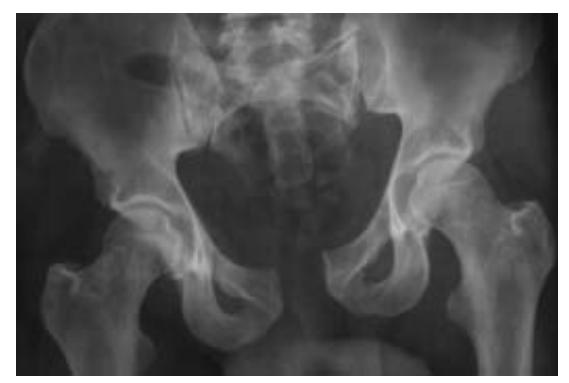

Abb. 3 Beckenübersicht mit instabiler Beckenringverletzung vom Typ C.

In allen anderen Situationen werden die therapeutischen und diagnostischen Maßnahmen entsprechend des allgemeinen Polytraumaalgorithmus durchgeführt. Die Schocktherapie wird mit mindesten 31 Kristalloiden innerhalb der ersten 10 Minuten ergänzt. Im gleichen Zeitrahmen erfolgt die Basisdiagnostik zum Ausschluss von Massenblutungen, bestehend aus klinischem Check, Röntgen des Thorax a.p., der Ultraschalluntersuchung des Abdomens und der Beckenübersichtsaufnahme (Abb.3).

Ist damit bis etwa 10-15 Minuten nach der Einlieferung der Kreislauf stabilisierbar, wird die Diagnostik erweitert. Der Zeitpunkt eventueller operativer Interventionen am Beckenring orientiert sich dann am allgemeinen Behandlungskonzept.
Bei weiter bestehendem instabilen Kreislauf wird die Massivtransfusion von notfalls ungekreuztem Blut eingeleitet, der Beckenring durch Zug und manuelle Kompression reponiert und eine Notfallstabilisierung des Beckenrings schon im Schockraum durchgeführt. Insbesondere bei Translationsverletzungen mit kompletter Lösung einer Beckenhälfte (Verletzungen des Typ C), hat sich die Beckenzwinge nach Ganz bewährt [3], alternativ wird eine Notfallstabilisation mit dem Fixateur externe durchgeführt. Bei Außenrotationsverletzungen des Typ B wird schon im Reanimationsraum ein einfacher ventraler Fixateur externe angelegt, um den Beckenring zu „schließen“.

\section{Technik der Anlage der Notfallbeckenzwinge}

Die Notfallzwinge sollte möglichst als steriles Komplettset im Reanimationsraum einsatzbereit gelagert werden. Die Orientierungspunkte im Beckenbereich des Verletzten können verletzungsbedingt unklar sein.

Die Notfallbeckenzwinge dient der Kompression des hinteren Beckenringes zur Erhöhung der mechanischen Stabilität und Reduktion des Blutverlustes.

Bestehen Unsicherheiten über die anatomischen Beziehungen, muss der Einsatz

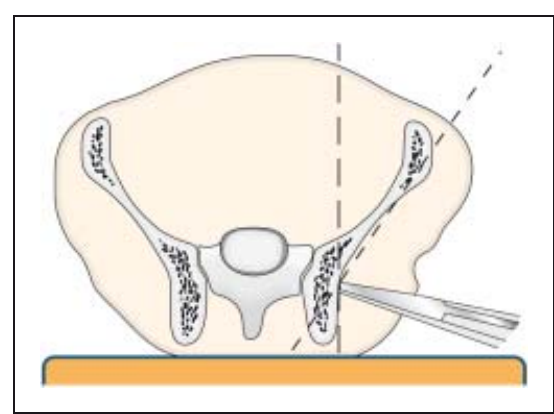

Abb.4 Die Flächenorientierung der Außenseite des Osilium ändert sich in Höhe der SIGelenke. Dadurch entsteht eine „Grube“, die als Orientierungshilfe des Spindeleintrittspunktes genutzt werden kann.

der Verankerungsdorne im Bildwandler kontrolliert werden, bevor die Zwinge eingesetzt wird!

Bei starker Fehlstellung wird die Reposition durch manuellen Zug am Bein, Innenrotation und ggf. lateralen, Kompression verbessert und manuell gehalten, bis die Beckenzwinge anliegt.

Zur Identifikation der Eintritts- und Ansatzpunkte erfolgt die Inzision an der Kreuzung zwischen Verlängerung der Femurachse über die Trochanterspitze und der Senkrechten von der Spina iliaca anterior superior. Bei Schwierigkeiten erfolgt die Orientierung mit Bildwandler. 


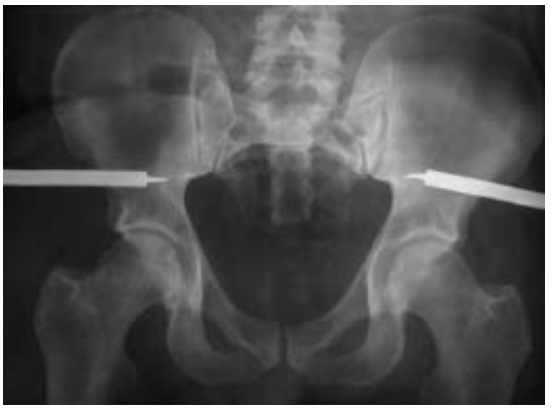

Abb. 6 Nach vollständiger Anlage erfolgt obligatorisch eine Röntgenkontrolle.

Da sich die Flächenorientierung der $\mathrm{Au}-$ ßenseite des Osilium in Höhe der SI-Gelenke ändert entsteht dadurch eine „Grube", die in Notfallsituationen als relativ sichere Orientierungshilfe genutzt werden kann (Abb.4). Die Beckenzwinge muss zur sicheren Verankerung möglichst in Höhe der SI-Gelenke platziert werden. Durch Palpation mit einem stumpfen Instrument, wie z.B. einer Klemme, ist diese Stelle auch bei stark angeschwollenem Weichteilmantel gut zu identifizieren.

Zur Montage der Beckenzwinge wird zunächst ein Spannarm eingeführt, dessen Spitze eingeschlagen und durch einen Assistenten fixiert wird. Danach wird der zweite Spannarm eingebracht und das Schienenteil eingeschoben. Durch manuelle Kompression an den Spannarmen wird die Beckenzwinge engültig fixiert durch Andrehen der Spindeln, bis es zur Vorbiegung des Schienenteils kommt (Abb.5).

Nach vollständiger Anlage erfolgt obligatorisch eine Röntgenkontrolle (Beckenübersicht a.p.), ggf. kann das Schienenteil zum besseren „handling“ verkürzt werden (Abb.6). Abschließend werden die Steinmann-Nägel abgepolstert. Bei ausgedehnten Inzisionen ist ggf. eine adaptierende Hautnaht notwendig.

Ist damit der Kreislauf 20-30 Minuten nach Einlieferung stabilisierbar, wird nach dem allgemeinem Polytraumaprotokoll weiterverfahren.

Bleibt der Kreislauf, bedingt durch die Beckenblutung, instabil oder ist er nur unter Massivtransfusionen stabil zu halten, wird die operative Blutstillung eingeleitet und der Patient unverzüglich in den Operationssaal gebracht.

Hier erfolgt nun die lokalisationsabhängige Versorgung der Blutung. Die Lage-

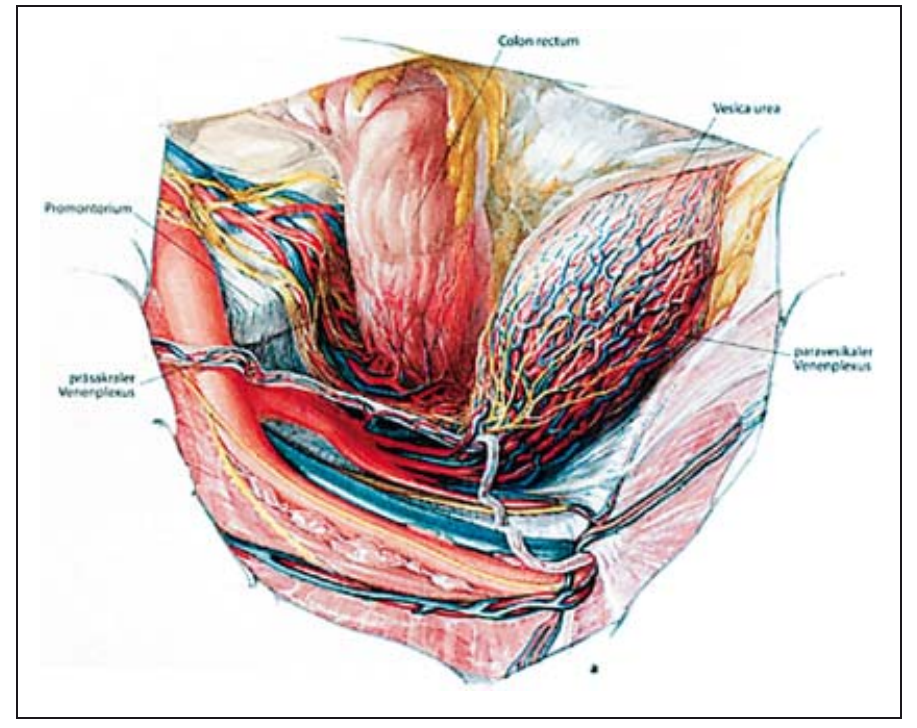

Abb. 7 Hauptblutungsquellen sind die präsakralen sowie paravesikalen Venenplexus.

rung zum Eingriff erfolgt in Rückenlage auf einem Standardoperationstisch. Eine Wärmematte sollte verwendet werden, um die einsetzende Hypothermie zu minimieren. Die Extremitäten, wenn möglich auch der Thorax, werden gegen Wärmeverluste mit Tüchern oder Goldfolie abgedeckt. Das Abdomen wird von der Symphyse bis zum Xyphoid in Zweifelsfällen auch inkl. des Thorax steril abgewaschen und abgedeckt.

Die Wahl der Inzision hängt vom sonographischen Befund ab. Sind keine oder nur geringe Mengen intraperitonealer Flüssigkeit sonographisch erkannt worden, wird eine mediane Hautinzision von der Symphyse bis knapp unterhalb des Nabels durchgeführt. Liegen große Mengen bzw. schnell zunehmende Mengen an intraperitonealer freier Flüssigkeit vor, wird eine mediane Laparotomie durchgeführt. Die Schnittführung sollte dann ausgedehnt und in der Regel vom Xyphoid bis zur Symphyse reichen.

Die Versorgung von intraperitonealen Organverletzungen erfolgt nach allgemeinen chirurgischen Regeln. Das Hauptziel sollte die möglichst schnelle Blutstillung sein. Bestehen simultan mehrere „Massenblutungen“, wird zunächst überall tamponiert, bei schwersten Blutungen aus dem Beckenraum die Aorta komprimiert oder vorübergehend ausgeklemmt. Nachdem Übersicht gewonnen wurde, wird die Versorgung der Organverletzungen schrittweise angegangen. Aufwendige rekonstruktive Verfahren im Abdomen sind in Notfallsitutationen, bei denen zusätzliche Blutungen aus dem Beckenbereich bestehen, nicht angezeigt.
Abdominelle Begleitverletzungen werden initial zunächst tamponiert.

Bei Milzverletzungen größeren Ausmaßes ist somit in der Regel eine Splenektomie nicht zu vermeiden, bei Leberverletzungen wird nach gezielter Versorgung großer Gefäße in der Regel auf die Tamponade zurückgegriffen, Darmverletzungen werden ausgeklemmt und abgestopft und erst nach Kontrolle der hämodynamischen Situation definitiv versorgt.

Liegt keine sonographisch nachweisbare intraperitoneale Blutung vor, wird die mediane Inzision lediglich vom Nabel bis zur Symphyse durchgeführt. Nach Durchtrennung der Faszie in der Linea alba stößt man insbesondere bei den $\mathrm{Au}$ ßenrotationsverletzungen oder bei Verletzungen des Typs $\mathrm{C}$ auf eine große Hämatomhöhle, aus der es erheblich blutet. Durch die komplette Zerreißung der Kompartmentgrenzen ist es möglich mit der Hand zumindest einseitig, oft auch beidseits die Blase zu umgreifen und direkt bis in den präsakralen Bereich vorzustoßen.

Hauptblutungsquellen im kleinen Becken sind der paravesikale und praesakrale Venenplexus.

Die Bereiche des präsakralen sowie des paravesikalen Venenplexus sind durch die Verletzung erheblich traumatisiert, die venösen Plexus sind weit aufgerissen (Abb.7). In dieser Situation ist gewöhnlich primär keine Übersicht zu gewinnen. Es wird zunächst eine Tamponade mit Bauchtüchern durchgeführt. Dazu wer- 

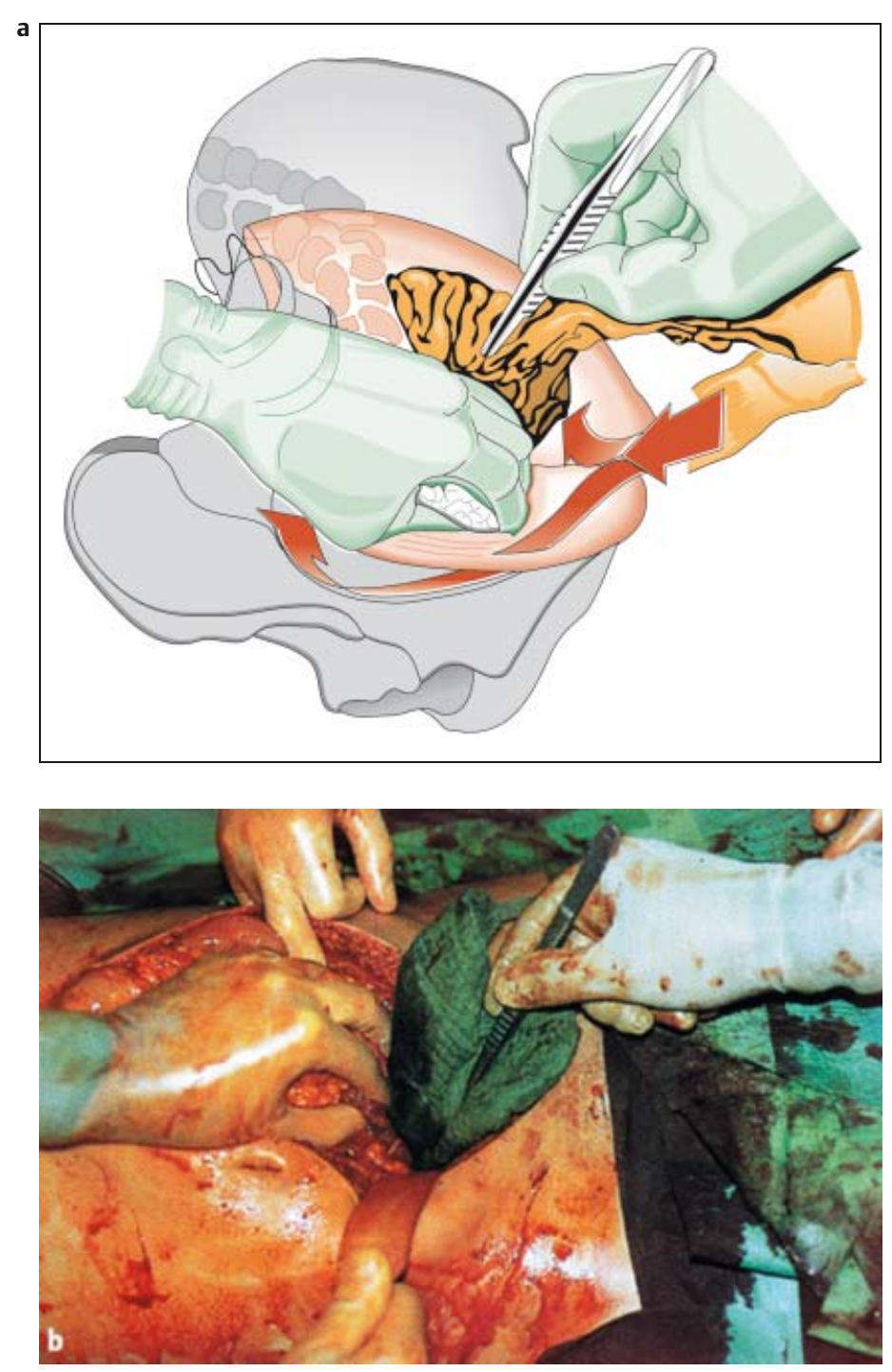

den jeweils 2-3 Bauchtücher oder große Rollen rechts und links der Blase eingelegt (Abb.8a u.b). Diese werden unter manueller Kontrolle gezielt mit der Pinzette schichtweise dorsal beginnend eingestopft. Die Tamponade ist bei korrekter Applikation auch in der Lage arterielle Blutungen zu unterdrücken, vor allem aber den Blutzustrom in das kleine Becken wesentlich zu verringern.

Nach kurzer Wartezeit werden zunächst die Tücher einer Seite entfernt, um unter jetzt gezielter Einstellung des Wundgebietes einen verbesserten Überblick zu gewinnen. Größere arterielle Blutungsquellen oder offensichtliche Gefäßstümpfe lassen sich so erkennen und gezielt verschließen. Bei dem überwiegenden Teil der Patienten verbleiben aber teilweise erhebliche, nicht lokalisierbare venöse Blutungen. Durch erneute gezielte Tamponaden werden diese kontrolliert.
Abb. 8 a u. b

Es wird zunächst eine Tamponade mit Bauchtüchern durchgeführt. Dazu werden jeweils 2-3 Bauchtücher oder große Rollen rechts und links der Blase sowie bis zum Sakrum eingelegt.

Bei Innenrotationsverletzungen ist es teilweise nötig die Beckenhälften vorsichtig manuell zu lösen, um eine ausreichende Übersicht und Zugangsmöglichkeit zu gewinnen. Danach werden auch hier die Tamponaden in oben beschriebener Weise eingelegt.

Bestehen Zerreißungen der großen Gefäße (A/V. iliaca communis, externa oder Art. femoralis), werden zunächst die Rupturstellen unter manueller Kompression des proximalen Gefäßstumpfes freipräpariert und ausgeklemmt. Die Entscheidung ob eine Gefäßrekonstruktion sinnvoll ist, hängt im Wesentlichen vom Ausmaß der pelvinen Verletzung ab. Ist der lumbosakrale Nervenplexus ausgerissen und besteht eine ausgedehnte Weichteilzerreissung, kann eine Gefäßrekonstruktion das Leben des Patienten gefährden. Besteht ein begrenzter Weichteilschaden und ist der Nervenplexus intakt wird die
Gefäßrekonstruktion (Naht, häufiger Interponat) durchgeführt, um die Extremität zu retten.

Wurde bei der manuellen Austastung eine größere Stufenbildung im Bereich des knöchernen hinteren Beckenringes bemerkt, so wird die zuvor angelegte Beckenzwinge durch einen Helfer gelöst und die Reposition durch Längszug am Bein und direkte Manipulation am Os ilium optimiert. Danach wird die Zwinge wieder fest angezogen. Nach dieser Korrektur muß ggf. die Tamponade nochmals optimiert werden.

Eine Verletzung im vorderen Beckenring wird abschließend durch eine einfache Osteosynthese stabilisiert. Bei Symphysenrupturen erfolgt eine Plattenosteosynthese, ggf. nur mit einer 2-Loch-Platte. Bei alleinigen transpubischen Instabilitäten erfolgt die Stabilisierung durch einen einfachen ventral supraacetabulär eingebrachten Zuggurtungsfixateur. Stark dislozierte Fragmente („Schambeinstachel") werden manuell reponiert und bei Redislokationstendenz durch $\mathrm{Mi}$ nimalosteosynthese gehalten (z. B. Spickdraht). Bei der Kombination Symphysenruptur und Scham- und Sitzbeinfrakturen wird durch Palpation entlang der Linea terminalis überprüft, ob eine echte transpubische „Instabilität“ vorliegt oder ob durch noch anhängendes Periost bzw. die bestehenden Bandverbindungen eine ausreichenden Knochenschienung besteht. In diesem Fall muss keine zusätzliche Stabilisierung des Schambeinastes erfolgen, der „Schluss“ der Symphyse ist ausreichend.

Die Stabilisierung der Verletzung des hinteren Beckenrings wird bei schlechtem Allgemeinzustand allein durch die Beckenzwinge vorgenommen, in Einzelfällen kann bei guter Stabilisierung des Allgemeinzustandes des Patienten im Rahmen der Primäroperation eine Verplattung des SI-Gelenkes von ventral vorgenommen werden. Sakrumfrakturen werden bei komplexen Beckentraumen aufgrund der anspruchsvollen Operationstechnik in der Notfallsituation nicht intern stabilisiert. Hier verbleibt immer die Beckenzwinge, aushilfsweise der Fixateur externe, bis der Allgemeinzustand des Patienten verlässlich stabilisiert werden konnte.

Bei den meist ausgekühlten Patienten kommt es häufig zu einem weiteren Durchsickern von Blut durch die Tamponade. Eine langwierige, den Patienten 
durch zusätzliche Blutverluste und Abkühlung weiter gefährdende, in der Regel sogar frustrane Suche nach einzelnen Blutungsquellen sollte unterbleiben. Gegebenenfalls wird die Tamponade nochmals erneuert und ihre Effektivität durch teilweisen Verschluss der Abdominalfaszie bis zum Nabel verbessert.

Liegen intraperitoneale Verletzungen vor, so werden diese zum jetzigen Zeitpunkt endgültig versorgt. Die Effektivität der zuvor durchgeführten Blutstillung im Bereich von Leber und Milz wird kontrolliert. Darmverletzungen werden endgültig versorgt oder eine ableitende Kolostomie angelegt.

Bei vorliegenden perinealen Verletzungen wird die Indikation zur Anlage eines doppelläufigen Anus praeter großzügig gestellt.

Die Indikation zur Anlage eines Anus pareter ist bei perinealen Verletzungen großzügig zu stellen.

Dabei ist zu beachten, dass dieser möglichst beckenfern, in der Regel als linksoder rechtsseitiger Anus praeter transversalis anzulegen ist, um eine sekundäre Kontamination der Beckenwunden zu vermeiden. Der abführende Schenkel wird ganz zum Abschluss des Eingriffs ausgespült („prograder wash out“) .

Das Abdomen bleibt in der Regel offen, um nicht durch die schwellungsbedingte, massive, intraperitoneale Druckerhöhung die Ventilationssituation des Patienten zu verschlechtern und das Risiko von druckbedingten, intraperitonealen Durchblutungsstörungen einzugehen („abdominelles Kompartmentsyndrom“). Der Verschluss erfolgt passager durch Abdecken der Darmschlingen und des großen Netzes mit feuchten Bauchtüchern. Nach Auflage einer Drainage auf die Bauchtücher wird eine große Inzisionsfolie luft- und wasserdicht aufgeklebt. Unter leichtem Dauersog wird ein „Durchnässen“ und damit weitere Auskühlung vermieden. Alternativ erfolgt der Bauchdeckenverschluss durch Einlage oder Annaht eines resorbierbaren Netzes („Vicrylnetz“) oder eines Reißverschlusses („Ethizip“).

Einem drohenden „abdominellen Kompartmentsyndrom " wird durch ein Abdomen apertum ohne Faszienschluß entgegenwirkt.
Nach der Akutversorgung wird der Patient möglichst umgehend auf die Intensivstation gebracht und aufgewärmt. Dazu haben sich neben Wärmematten insbesondere „Warmluftdecken“ bewährt. Es ist auf alle Fälle darauf zu achten, dass der Patient trocken liegt, um weitere Wärmeverluste $\mathrm{zu}$ vermeiden. Ein eventueller Sekretaustritt aus den Wunden muss durch seitlich am Patienten angeklebte wasserundurchlässige Folien vom Körper abgeleitet werden. Anderenfalls sind die Wärmeverluste nicht zu kompensieren.

Besteht trotz dieser Maßnahmen weiterer Transfusionsbedarf, sollte, wenn vertretbar, die Aufwärmung abgewartet werden, bevor erneute interventionelle Maßnahmen ergriffen werden. Meist sistieren die Blutverluste mit Erwärmung spontan.

Bei nicht tolerierbaren Blutverlusten wird je nach Einzelbefund entschieden, ob eine erneute Revision und Tamponadenoptimierung angeschlossen wird oder bei effektiver Tamponade eine Angiographie mit evtl. selektiver Embolisation vorzuziehen ist.

Bei stabiler Hämostase wird für 24 Stunden, besser 48 Stunden, zugewartet und danach eine geplante Tamponadenentfernung durchgeführt.

Ein geplanter Tamponadenwechsel erfolgt nach 48 Stunden.

In der Regel lassen sich jetzt noch kleinere arterielle oder venöse Blutungsquellen gezielt unterbinden. Ist die Situation weiterhin unübersichtlich und nehmen die diffusen Blutungen nach Entfernung wieder zu, wird die Tamponade erneut eingelegt. Die Faszie wird erneut verschlossen und diese Revisionen im Intervall von 24 bis 48 Stunden so lange fortgeführt, bis Bluttrockenheit besteht.

\section{Ergebnisse}

Im eigenen Kollektiv wurden zwischen Juni 1989 und Juni 1998 von insgesamt 861 Patienten mit Beckenringverletzungen, 39 Patienten mit translationsinstabilen C-Verletzungen des Beckens mittels Notfallbeckenzwinge primär stabilisiert.

Das Durchschnittsalter betrug 36 Jahre (11 - 89 Jahre), 29 Patienten waren männlich, 10 weiblich. Die Unfallursache war in 80\% ein Unfall im Straßenverkehr, 15,4\% stürzten aus größerer Höhe ab. Zwei Patienten erlitten eine isolierte Beckenver- letzung, die übrigen waren mehrfachverletzt, 27 waren polytraumatisiert. Der mittlere Hannover Polytrauma-Score (PTS)[5] betrug 39,9 Punkte (11-79 Punkte), entsprechend Gruppe III. Per Definition wiesen alle Patienten eine Beckenringverletzung des Typ $C$ nach Tile auf.

Der primäre Hämoglobinwert bei Eintreffen in der Notaufnahme betrug durchschnittlich $6,7 \mathrm{~g} / \mathrm{dl}(3,1-12,3 \mathrm{~g} / \mathrm{dl})$, der durchschnittliche Base Exzess betrug $-8,7 \mathrm{mmol} / \mathrm{l}(+2--28,0 \mathrm{mmol} / \mathrm{l})$. Der durchschnittliche systolische Blutdruck betrug $82 \mathrm{~mm} \mathrm{Hg}(0-130 \mathrm{~mm} \mathrm{Hg})$. Diese Werte deuten auf ein erhebliches Schockgeschehen hin.

Die Indikation zur Anlage der Beckenzwinge war bei 13 Patienten die alleinige Beckeninstabilität. Bei den übrigen 26 Patienten bestand zusätzlich zur instabilen Beckenverletzung eine Kreislaufinstabilität. Bei 15 Patienten kam es bereits nach Anlage der Beckenzwinge bei gleichzeitiger Volumentherapie zu einer Stabilisierung der Kreislaufsituation, bei fünf $\mathrm{Pa}-$ tienten trat keine Veränderung ein, bei sechs Patienten verschlechterte sich die Kreislaufsituation trotz Anlage der Beckenzwinge und begleitender Volumentherapie. Bei 20 dieser Patienten wurde die Zwinge innerhalb von 30 Minuten nach Aufnahme angelegt, die durchschnittliche Applikationszeit betrug 60 Minuten.

Zur Beurteilung der Effektivität der Maßnahme Beckenzwinge und des eigenen etablierten Behandlungsalgorythmus für komplexe Beckentraumata, wurden die 14 Patienten analysiert, bei denen neben einem komplexen Beckentrauma eine instabile Beckenringverletzung mit begleitender Kreislaufinstabilität vorlag und die primär behandelt wurde (keine sekundären Verlegungen). Diese Patienten erhielten neben der Notfallstabilisierung zusätzlich eine Tamponade des kleinen Beckens zur peripelvinen Blustillung. Der primäre Hämoglobinwert bei Eintreffen in der Notaufnahme betrug in dieser Untergruppe durchschnittlich 5,6g/dl $(2,1-7,8 \mathrm{~g} / \mathrm{dl})$, der durchschnittliche Base Exzess betrug - 12,1 mmol/1 (-3$-28,0 \mathrm{mmol} / \mathrm{l})$. Der durchschnittliche systolische Blutdruck betrug $70 \mathrm{~mm} \mathrm{Hg}$ (0-95 mm Hg).

Zur Effektivitätsbeurteilung wurde die erwartete Letalität dieser Patienten mit der TRISS-Methode [2] bestimmt. Dabei ergab sich eine errechnete Überlebens- 
wahrscheinlichkeit von $22 \%$, gegenüber $43 \%$ tatsächlicher Überlebensrate.

\section{Schlussfolgerung}

Instabile Beckenringverletzungen vom Typ C mit gleichzeitiger Kreislaufinstabilität sind seltene Verletzungen, die nur in $1,6 \%$ aller Beckenringverletzungen auftreten. Die Kombination aus mechanischer Beckenringstabilisierung mittels Beckenzwinge und Kreislaufstabilisierung bei beckenbedingter Blutung mittels pelviner Tamponade hat sich dabei als effektive Maßnahme zur Stabilisierung der Gesamtsituation erwiesen.

\section{Literatur}

1 Bosch U, Pohlemann T, Haas N, Tscherne H. Klassifikation und Management des komplexen Beckentraumas. Unfallchirurg 1992; 95: 189-196

2 Boyd C, Tolson M, Copes W. Evaluating trauma care: The TRISS method. J Trauma 1987; 27: 370 - 378

3 Ganz R, Krushell R, Jakob R, Küffer J. The antishock pelvic clamp. Clin Orthop 1991; 267: $71-78$

${ }^{4}$ Huittinen V, Slätis P. Postmortem angiography and dissection of the hypogastric artery in pelvic fractures. Surgery 1973; 73: $454-$ 462

5 Oestern H, Tscherne H, Nerlich M. Klassifizierung der Verletzungsschwere. Unfallchirurg 1985; 88: $465-472$

6 Pohlemann T, Gänsslen A, Hartung S. für die Arbeitsgruppe Becken, H.:.T., H.: Beckenverletzungen/Pelvic Injuries. Hefte zu „Der Unfallchirurg“" 1997; Heft 266
7 Pohlemann T., et al.: Beckenverletzungen: Epidemiologie, Therapie und Langzeitverlauf. Übersicht über die multizentrische Studie der Arbeitsgruppe Becken. Unfallchirurg 1996; 99: 160 - 167

8 Tscherne H, Pohlemann T. Tscherne Unfallchirurgie: Becken und Acetabulum. Springer-Verlag, Berlin, 1998

Prof. Dr. med. T. Pohlemann

Ltd. Oberarzt

Dr. med. A. Gänsslen

Assistenzarzt

Unfallchirurgische Klinik Medizinische Hochschule Carl-Neuberg-Str. 1 30623 Hannover 